\title{
Prevención de riesgos laborales y desempeño de trabajadores del Instituto Continental, Huancayo
}

\author{
Prevention of occupational risks and performance of workers \\ of the Instituto Continental, Huancayo
}

Fabiola Berrios Gamarra ${ }^{1}$

1 Instituto Continental, Huancayo, Perú

\section{RESUMEN}

El objetivo fue determinar la efectividad de un programa de prevención de riesgos laborales sobre el desempeño laboral de los trabajadores de limpieza del Instituto Continental al 2016. El estudio fue descriptivo transeccional con pre y post intervención. La población fue de 35 trabajadores; para la recolección de datos se utilizó la técnica de la observación y el instrumento fue la ficha de evaluación de desempeño, se contrasto la hipótesis mediante la comparación de las medias empleando la prueba paramétrica de diferencia de dos medias para muestras relacionadas. Se tiene como resultados que, en cuanto a la evaluación del desempeño antes de la implementación del programa de riesgos laborales, el 74,3\% tienen un desempeño bueno; no hay evidencia de desempeño muy bueno. Después de la implementación del programa se observa que $65,7 \%$ de trabajadores tienen un desempeño bueno y el 5,7 \%, presentan un desempeño muy bueno. Sin embargo, no existe una relación significativa $(p>0,05)$ entre el desempeño laboral antes y después de la aplicación del programa. Las actividades teóricas y prácticas del programa de prevención de riesgos laborales no mejoraron significativamente las competencias generales como: identidad, iniciativa, integridad, así como las competencias específicas: cumplimiento de objetivos, eficiencia, trato al cliente, comunicación, adaptabilidad, confiabilidad, trabajo en equipo. En conclusión, la aplicación del programa de prevención de riesgos laborales no fue eficaz sobre el desempeño laboral de los trabajadores.

Palabras claves: Programa, prevención de riesgos laborales, desempeño, trabajador de limpieza.

\section{ABSTRACT}

The objective was to determine the effectiveness of a program of prevention of occupational risks on the job performance of the cleaning workers of the Instituto Continental in 2016. The study was descriptive transectional with pre and post. The population was 35 workers; the observation technique was used for the collection of data and the instrument was the performance evaluation form, the hypothesis by comparing averages is contrast using the parametic test of two difference for related samples. It is results which, in terms of the performance evaluation before the implementation of the program of occupational risks, $74,3 \%$ have a good performance; there is no evidence of very good performance. After the implementation of the program is observed that 65,7 $\%$ of workers have a good performance and 5,7\%, present a very good performance. However, there is no significant relationship $(p>0,05)$ betweeen job performance before and after the application of the program. The theoretical and practical activities of the occupational risk prevention program did not significantly improve the general skills such as: identify, initiative, integrity, as well as specific competences: compliance with objectives, efficiency, customer treatment, communication, adaptability, reliability, teamwork. In conclusion, the application of the occupational risk prevention program was not effective on the work performance of the workers.

Keywords: Program, prevention of occupational risks, performance, cleaning worker.

Historial del artículo:

Recibido, 01 de agosto de 2016; aceptado, 21 de junio de 2017; disponible en línea, 30 de junio de 2017

* Licenciada en Enfermería de la Universidad Nacional Mayor de San Marcos.

Correo: fberrios@continental.edu.pe 


\section{INTRODUCCIÓN}

La Oficina Internacional del Trabajo (OIT,2005) calculó que 2,2 millones de personas morían cada año en el mundo a causa de accidentes y enfermedades relacionados con el trabajo, cifra que parece ir en aumento. Además, cada año se producen unos 270 millones de accidentes de trabajo no mortales (que resultan en un mínimo de tres días de baja laboral) y 160 millones de casos nuevos de enfermedades profesionales. La seguridad y la salud en el trabajo es motivo de preocupación en todo el mundo para los gobiernos, los empleadores, y los trabajadores y sus familias.

El enfoque actual de la prevención de riesgos laborales se inscribe en la definición de la Organización Mundial de la Salud (OMS), que considera la salud no sólo como una ausencia de enfermedad sino también como el bienestar físico, mental y social de las personas entendiendo la prevención de riesgos laborales como la disciplina que busca promover la mejora de la seguridad y salud de los trabajadores mediante la aplicación de medidas y el desarrollo de las actividades necesarias para prevenir los riesgos derivados de las condiciones del trabajo, teniendo como herramienta fundamental la evaluación de riesgos desarrollada en cada empresa.

A los trabajadores siempre debería proporcionárseles: formación sobre medidas de protección, información periódica sobre los peligros concretos inherentes a sus trabajos, acceso a información sobre los peligros de tipo general a los que están expuestos en sus lugares de trabajo sobre: peligros físicos como el ruido, temperatura inadecuada o iluminación insuficiente, sustancias químicas y biológicas peligrosas y sus posibles efectos perjudiciales para la salud, factores psicosociales (OIT, 2013).

El análisis preliminar de riesgo, es el proceso dirigido a estimar la magnitud de aquellos peligros que no hayan podido evitarse, obteniendo la información necesaria para que el empresario esté en condiciones de tomar una decisión apropiada sobre la necesidad de adoptar medidas preventivas $y$, en tal caso, sobre el tipo de medidas que deben adoptarse.

\section{Programa de prevención de riesgos laborales}

El programa brindo información precisa y clara referente a los riesgos que puedan encontrar en el curso de su trabajo y las acciones necesarias para resolverlos, este entrenamiento fue dinámico y se amoldo a las circunstancias que se presentaron durante el desarrollo de sus tareas diarias. Tuvo sus propios objetivos como el de preservar la salud de los trabajadores en sus actividades de trabajo mediante la prevención de accidentes y enfermedades profesionales, promoviendo la salud en el espacio

Tabla 1

Resúmen global de riesgos.

\begin{tabular}{lc}
\hline Nivel de riesgo & Cantidad \\
\hline Bajo & 05 \\
Moderado & 04 \\
Alto & 08 \\
\hline
\end{tabular}

laboral.

El Programa de Prevención de Riesgos incorporo las siguientes áreas:

- Seguridad Laboral: Uso de extintores, luces de emergencia, simulacros. Análisis de riesgos (ergonómicos, higiénicos), Uso de Equipos de protección personal.

- Salud Ocupacional: Exámenes médicos, seguimiento, vigilancia.

- Salud General: Charlas preventivo promocionales, consejería en salud, Inmunizaciones.

Se realizaron charlas, talleres, entrevistas, consejería y vigilancia.

Tabla 2

Matriz general de riesgos de personal de limpieza.mètodos de evaluación RULA, OWAS, ISTAS.

\begin{tabular}{|c|c|c|c|c|c|c|}
\hline Puesto & Actividad & RULA & OWAS & ISTAS & JSI & Final \\
\hline \multirow{5}{*}{$\begin{array}{l}\text { Personal } \\
\text { de } \\
\text { limpieza }\end{array}$} & $\begin{array}{l}\text { Limpieza de sillas } \\
\text { y mesas }\end{array}$ & & & & & \\
\hline & Barrer el aula & & & & & \\
\hline & Trapear aula & & & & & \\
\hline & Levantamiento & & & & & \\
\hline & Transporte & & & & & \\
\hline
\end{tabular}




\section{Desempeño Laboral}

Chiavenato (2002) expone que el desempeño es "eficacia del personal que trabaja dentro de las organizaciones, la cual es necesaria para la organización, funcionando el individuo con una gran labor y satisfacción laboral". En tal sentido, el desempeño de las personas es la combinación de su comportamiento con sus resultados, por lo cual se deberá modificar primero lo que se haga a fin de poder medir y observar la acción. El desempeño define el rendimiento laboral, es decir, la capacidad de una persona para producir, hacer, elaborar, acabar y generar trabajo en menos tiempo, con menor esfuerzo y mejor calidad, estando dirigido a la evaluación la cual dará como resultado su desenvolvimiento.

Benavides (2002) relaciona desempeño con competencias, afirmando que en la medida en que el trabajador mejore sus competencias mejorará su desempeño. Para esta autora, las competencias son "comportamientos y destrezas visibles que la persona aporta en un empleo para cumplir con sus responsabilidades de manera eficaz y satisfactoria. Igualmente, expone que los estudios organizacionales se proyectan alrededor competencias fundamentales; estas competencias son: competencias genéricas (amplitud de conocimientos, astucia para tener un entendimiento claro, razonamiento para tener alternativas, organización para trabajar productivamente) , competencias laborales(conocimientos, habilidades y destrezas, rasgos y temperamentos, motivos y necesidades) y competencias básicas(escuchar, hablar, capacidad lectora, escritura, aritméticas y matemáticas, pensamiento creativo, solución de problemas, toma de decisiones, capacidad de aprender a razonar, asimilación y comprensión, autorresponsabilidad, autoestima, autodirección, integridad, sociabilidad; Competencias específicas, son la base particular del ejercicio profesional y están vinculadas a condiciones específicas de ejecución; se relacionan con la disciplina, son propias de cada profesión; permiten la compatibilidad entre los diferentes programas de una disciplina y la definición de cada profesión; son consecuencia de los conocimientos y las habilidades adquiridos a través de un programa educativo: resultado de aprendizaje.

Martínez (2011) estudia el proceso de gestión de la seguridad basado en los comportamientos determina las variaciones que deben producirse en los comportamientos de los colaboradores cuando los supervisores cambian sus actitudes y nivel de desempeño orientado a la seguridad. Busca corroborar la hipótesis de que un mejor quehacer de los supervisores con respecto a sus comportamientos en el tema de la seguridad producirá un mejoramiento positivo en conductas seguras de los operarios y colaboradores. A partir de esta información fue posible indicar las formas de modificar comportamientos y responsabilidades en los supervisores, analizando de forma paralela el efecto que ello producía en los comportamientos de los colaboradores hacia la seguridad este último medido por medio del indicador: comportamientos seguros.

Villalobos (2011) estudia la perspectiva clínica de la consciencia del riesgo en la accidentalidad laboral con el objetivo de analizar el fenómeno de la accidentalidad laboral en un grupo de 10 trabajadores de una empresa productora de papel, cuyas acciones conllevaron a un accidente incapacitante. Utilizó una entrevista semi-estructurada y se analizó la información siguiendo el enfoque cualitativo de tipo constructivo-interpretativo. El análisis comprensivo de las entrevistas permitió identificar que, aunque en la empresa se siguieran los lineamientos del Sistema General de Riesgos Profesionales en Colombia, los trabajadores accidentados tuvieran el conocimiento completo de los riesgos y el deseo de protegerse, no lograron articular estos aspectos para dirigir sus acciones en el momento de la situación de riesgo. Los resultados evidenciaron que el problema se ubica en el nivel de la conciencia de sí mismo como valor de vida y no en el de la cognición como conocimiento o información.

Aguirre (2002) plantea la influencia de un programa educativo en el nivel de conocimientos de enfermedades ocupacionales, el procesamiento de datos se realizó con la aplicación de la prueba estadística de Mc Nemar encontrando que la aplicación de un programa educativo incremento significativamente el nivel de conocimientos sobre enfermedades ocupacionales en transportistas lo cual se ve reflejado en los resultados que demuestran que el nivel de conocimientos aceptable, incrementó significativamente luego de aplicado el programa educativo.

La presente investigación se justifica porque al aplicar un programa de prevención de riesgos se reconoce, respeta y se pone en práctica la Ley № 29783, Ley de Seguridad y Salud en el Trabajo que tiene por objetivo promover una cultura de prevención de riesgos laborales a través del deber de prevención de los empleadores, estamos brindando servicios de prevención en el ámbito educativo a través de evaluaciones de riesgo dentro de la misma institución. Al realizar la evaluación de dicho puesto de trabajo, garantizamos la eliminación de los daños continuados a la salud del trabajador.

Resulta beneficioso, tanto para el empleador como para el trabajador, y esto puede reflejarse en: la evolución y desarrollo del trabajador, elevada motivación, mejor desenvolvimiento de sus funciones (desempeño), menor rotación en el empleo, menores tasas de 
ausentismo, menos quejas, mayor satisfacción en el empleo. Menos accidentes del trabajo, prevención de enfermedades profesionales y relacionadas con el trabajo.

El objetivo general de la investigación fue determinar la efectividad de un programa de prevención de riesgos laborales sobre el desempeño laboral de los trabajadores de limpieza del Instituto Continental Huancayo.

Los objetivos específicos fueron: Identificar y analizar el desempeño laboral de los trabajadores de limpieza del Instituto Continental antes de la aplicación del programa de prevención de riesgos laborales.

Demostrar que las actividades teóricas y prácticas del Programa de prevención de riesgos laborales mejoran las competencias generales y específicas de los trabajadores de limpieza del Instituto Continental. Identificar y analizar el desempeño laboral de los trabajadores de limpieza del Instituto Continental después de la aplicación del programa de prevención de riesgos laborales.

\section{MATERIAL Y MÉTODOS}

La investigación se realizó utilizando el enfoque cuantitativo, teniendo en cuenta los objetivos de la investigación, el diseño fue pre experimental - con pre y post intervención- transeccional descriptivo.

La población de estudio en la investigación fue de 35 trabajadores, quienes participaron en el desarrollo de todo el programa de prevención de riesgos y su desempeño fue evaluado por el área de gestión humana de dicha institución.

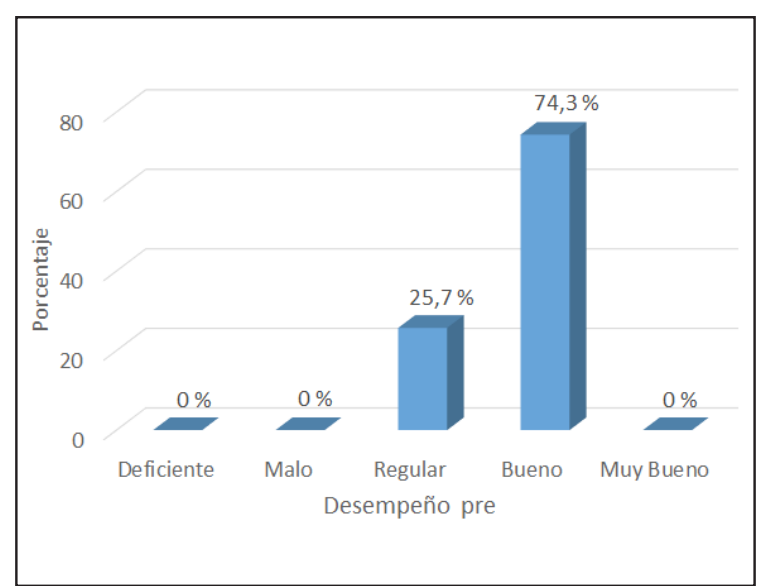

Figura 2.Desempeño laboral de los trabajadores antes de la aplicación del programa de prevención de riesgos laborales.

\section{Técnicas e instrumentos}

Se utilizó el análisis documental: que sirvió para analizar sistemática y objetivamente los documentos producidos (documentos oficiales, historias clínicas textos, entre otros). También se aplicó la técnica de la observación y como instrumento el formato de evaluación del desempeño utilizado por el área de gestión humana de la organización. Esta evaluación del desempeño se mide a través de pruebas de $360^{\circ}$, que según Martha Alles "es un sistema de evaluación sofisticado" (Chiavenato,2002).

El propósito de aplicar la evaluación de 360 grados es darle al empleado la retroalimentación necesaria para tomar las medidas para mejorar su desempeño, su comportamiento o ambos, y dar a la gerencia la información necesaria para tomar decisiones en el futuro. La oficina de gestión humana elaboró una ficha de evaluación de desempeño basada en competencias la cual fue aplicada en el mes de marzo de 2016, en un primer momento y luego de la implementación del programa de prevención de riesgos, en el mes de noviembre de 2016.

Esta ficha consta de: competencias generales: identidad, iniciativa, integridad, cooperación, y competencias específicas: cumplimiento de objetivos, eficiencia, trato al cliente, comunicación, adaptabilidad, confiabilidad, trabajo en equipo, desarrollo profesional.

\section{RESULTADOS}

En cuanto a la evaluación del desempeño de los trabajadores antes de la aplicación del programa de riesgos laborales, se observa que del $100 \%$ de evaluados, 74,3 \% tienen un desempeño bueno mientras que el $25,7 \%$ tienen un desempeño regular.

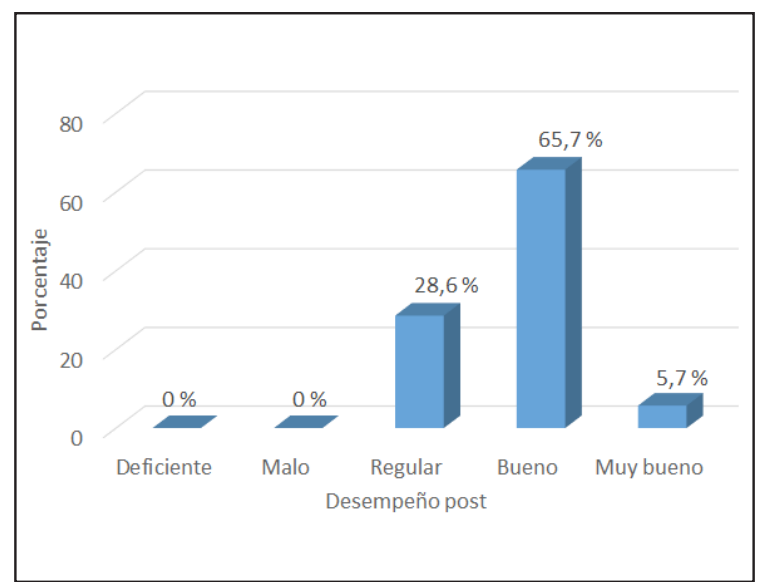

Figura 3.Desempeño laboral de los trabajadores después de la aplicación del programa de prevención de riesgos laborales, Muy Bueno (5,7\%). 
Según la evaluación de desempeño por competencias genéricas tenemos que el 74,3 \% trabajadores tiene un desempeño bueno; 22,9\%, tiene un desempeño regular. En la evaluación por competencias específicas, tenemos que el $68,6 \%$ trabajadores tiene un desempeño bueno; $25,7 \%$, tiene un desempeño regular.

En cuanto a la evaluación del desempeño de los trabajadores después de la implementación del programa de riesgos laborales, se observó que de un total de $100 \%$ de evaluados: $65,7 \%$ trabajadores tienen un desempeño bueno; el 28,6 \% regular y el 5,7 $\%$ tienen un desempeño muy bueno.

Según la evaluación de desempeño por competencias genéricas después de la implementación del programa de prevención de riesgos laborales, tenemos que el $74,3 \%$ trabajadores tiene un desempeño bueno; $17,1 \%$, tiene un desempeño regular y $8,6 \%$ tiene un desempeño muy bueno. Según la evaluación de desempeño por competencias específicas, después de la implementación del programa de prevención de riesgos laborales, tenemos que el 65,7 \% trabajadores tiene un desempeño bueno; $28,6 \%$, tienen un desempeño regular y $5,7 \%$ tienen un desempeño muy bueno.

\section{DISCUSIÓN}

Teniendo en cuenta el derecho a la salud integral del trabajador, la relación existente entre salud laboral y calidad de vida laboral y toda la normativa vigente en materia de salud laboral es necesario establecer servicios de prevención de tal manera que a través de ellos se realicen las evaluaciones de riesgo, la evaluación de cada puesto de trabajo, la vigilancia específica de la salud garantizando la disminución de los daños continuados a la salud que pueden deteriorar a la persona. Esto se logra a través de la implementación de proyectos prevención de riesgos laborales puede resultar beneficiosa, tanto para la organización como para el trabajador, y reflejándose en: la evolución y desarrollo del trabajador, elevada motivación, mejor desenvolvimiento de sus funciones (desempeño), menor rotación en el empleo, menores tasas de ausentismo, menos quejas, tiempo de ocio reducido, mayor satisfacción en el empleo, mayor eficiencia en la organización, menos accidentes del trabajo, prevención de enfermedades relacionadas con el trabajo.

La implementación del programa de prevención de riesgos laborales como conjunto de actividades preventivas que evitan o disminuyen la probabilidad de que la exposición a un factor o proceso peligroso en el trabajo cause enfermedad o lesión, se encuentra dentro de las actividades que involucran la seguridad y salud en el trabajo, la cual según la nueva normativa es establecida como el primer principio de la ley, esta menciona la posición de garante del empleador ,pues este garantiza, en el centro de trabajo,el establecimiento de los medios y condiciones que protejan la vida, la salud y el bienestar de los trabajadores (Ley SST,2011).

Según Paredes (2013), asesora legal de soluciones laborales, se debe hacer una crítica a lo dispuesto en la ley como principio de prevención, toda vez que se exime de responsabilidad alguna al trabajador respecto de este deber. Entiéndase que como está dispuesto por las normas generales en seguridad y salud, la obligación es tripartita: estado, empleador, trabajador, por lo que uno de los objetivos del presente programa implementado fue lograr que el personal desarrolle una cultura preventiva en sus actividades laborales, a través de su autocuidado.

En esta línea de ideas, basándonos en el trabajador, si este no cumple con las disposiciones de los especialistas en materia de prevención de riesgos, no serviría de mucho la aplicación del mejor sistema de seguridad y salud, vemos que para ello intervienen una serie de factores tales como aptitud, actitud, compromiso con la gestión de seguridad y salud, formación o capacitación; los cuales cuentan con indicadores de evaluación ,motivo por el cual se evaluó el desempeño laboral del trabajador esperando observar dentro de las competencias evaluadas, cambios, después de implementar el programa de prevención de riesgos, el resultado es que la implementación del programa no tiene un efecto significativo en el desempeño laboral de los trabajadores de limpieza de la institución educativa. Siendo asi nuestros resultados guardan relación con la investigación de Villalobos (2011) acerca de la Perspectiva clínica de la consciencia del riesgo en la accidentalidad laboral sus resultados evidenciaron que el problema se ubica en el nivel de la conciencia de sí mismo como valor de vida y no en el de la cognición como conocimiento o información.

Los resultados obtenidos difieren a los hallazgos de Mayuri B.(2006), en Lima, demuestra que un programa de de capacitación mejora significativamente el desempeño laboral de los participantes, permitiendo introducir cambios en la cultura para reorientar a la organización hacia la Excelencia (desempeño) con la sociedad de la que toma recursos y a la que sirve.

La evaluación del desempeño basado en competencias permite observar al trabajador en la medida que mejore sus competencias mejorará su desempeño. Para Benavides $O$. las competencias son comportamientos y destrezas visibles que la persona aporta en un empleo para cumplir con sus responsabilidades de manera eficaz y satisfactoria. Según los resultados presentados las competencias que demuestran una tendencia a cambio son: identidad, la cooperación y la confiabilidad. 
Sin embargo según Martínez (2011) el diseño de nuevos indicadores y formas proactivas de evaluación de desempeño, da un mayor alcance para medir el desarrollo o consistencia del proceso de seguridad basado en los comportamientos, garantiza una mayor efectividad y eficiencia general del mismo, llegando a la conclusión de que si logran un mejor desempeño en sus comportamientos hacia la seguridad, se logrará un mejoramiento positivo de los comportamientos de los operarios o colaboradores y se lograrán aumentar aquellos considerados seguros.

En conclusión la aplicación de un programa de prevención de riesgos laborales no fue eficaz en la mejora del desempeño laboral de los trabajadores de limpieza del Instituto Continental.

\section{REFERENCIAS \\ BIBLIOGRÁFICAS}

Mayurí B.J. (2008) Perù:Capacitación empresarial y desempeño laboral en el Fondo de Empleados del Banco de La Nación - FEBAN [tesis doctoral].

Martínez O. C.(2011) Colombia: El proceso de gestión de la seguridad basado en los comportamientos.

Villalobos, M.y Zuñiga,W.(2011)Colombia: Perspectiva clínica de la consciencia del riesgo en la accidentalidad laboral: un estudio cualitativo.
Aguirre C. K., (2002) Perù:Influencia de un programa educativo en el nivel de conocimientos de enfermedades ocupacionales.

Chiavenato, I.(2002)Mèxico: Gestión del Talento Humano. McGraw - Hill

MINTRA (2011)Perù:Ley de seguridad y salud en el trabajo, 29783 DS 005 ElPeruano,448694,20 agosto.

Fundación Mapfre Manual de Higiene Industrial España: Ediciones Mapfre

OIT.com, (2013):Seguridad y salud en el trabajo[sede Web]. Organización Internacional del Trabajo, 1996 - actualizada 12 de marzo 2013; acceso 15 de mayo de 2013.Disponible en http://www.ilo.org/ global/topics/safety-and-health-at-work/lang--es/ index.htm

Benavides, O.(2002) Colombia: Competencias y Competitividad. Diseño para Organizaciones Latinoamericanas. Bogotá: McGraw - Hill.

Paredes E. B.(2013 Soluciones Laborale: disponible en:http://worldcat.org/identities/Iccnno2013038639/

INSHT(2011)España:Evaluación de Riesgos Laborales disponible en :http://www.insht.es/InshtWeb/ Contenidos/Documentacion/TextosOnline/Guias Ev_Riesgos/Ficheros/Evaluacion_riesgos.pdf 